as much as the least active RA patients, it is difficult for activities in daily life, that the quality of life associated with the illness is low, and that depression and anxiety are similar to employees. As a result, disease activity, quality of life and functional status control in RA patients are as important as those who are working as housewives. Particularly in increasing productivity and participation in everyday life, the mood is influential and physicians must examine their patients in this regard. There is a need for more extensive cohort studies on this topic. Disclosure of Interest: None declared

DOI: 10.1136/annrheumdis-2017-eular.4614

\section{AB1203-HPR COMPARISON OF QUALITY OF LIFE OF PATIENTS WITH RHEUMATOID ARTHRITIS, PSORIATIC ARTHRITIS AND ANKILOSING SPONDILITIS WITH TREATMENT OR PRESCRIPTION OF BIOLOGIC DRUGS: RESULTS FROM THE CARA STUDY}

L. Sinigaglia ${ }^{1}$, L. Scalone ${ }^{2,3}$, P. Sarzi-Puttini ${ }^{4}$, C.M. Montecucco ${ }^{5}$ R. Giacomelli ${ }^{6}$, G. Lapadula ${ }^{7}$, I. Olivieri ${ }^{8}$, A. Giardino ${ }^{9}$, G. Didoni $^{9}$ P. Cortesi $i^{2,3}$, L.G. Mantovani ${ }^{2,3}$, M. Mecchia ${ }^{9}$ on behalf of CARA study working group. ${ }^{1}$ Rheumatology Unit, G. Pini Hospital; ${ }^{2}$ CHARTA Foundation, Milano; ${ }^{3}$ University of Milano Bicocca, Monza; ${ }^{4}$ Rheumatology Unit, L. Sacco University Hospital, Milano: ${ }^{5}$ University of Pavia School of Medicine, IRCCS Policlinico San Matteo Foundation, Pavia; ${ }^{6}$ Rheumatology Unit School of Medicine, University of L'Aquila, L'Aquila; ${ }^{7}$ Rheumatology Unit, University of Bari, Bari; ${ }^{8}$ San Carlo Hospital of Potenza and Madonna delle Grazie Hospital of Matera, and the Basilicata Ricerca Biomedica (BRB) Foundation, Italy, Potenza and Matera; ${ }^{9}$ MSD Italia, Roma, Italy

Background: Chronic rheumatic conditions such as rheumatoid arthritis (RA), ankylosing spondylitis (AS), and psoriatic arthritis (PsA) are associated with severe morbidity and significant impairment of patients' health related quality of life (HRQoL). Several treatments are available but not all the patients respond positively to them. Biologic therapies such as anti-TNF $\alpha$ agents are shown to benefit who fail or have partial responses to standard DMARD therapy.

Objectives: Within a multicenter stated preferences study (CARA Study), we assessed HRQoL in patients with RA, AS and PsA, and estimated relationship of $H R Q O L$ with the different diagnoses, clinical characteristics and biological treatment experience.

Methods: Patients with RA, AS, or PsA, who at the time of enrollment were following a treatment (experienced) or received a first prescription (naïve) of treatment with biological drugs were enrolled. Together with preferences data, clinical and HRQOL information was reported. HRQoL was assessed with the recently developed and successfully validated version of the EQ-5D-5L, which allows to obtain a description of health (in 5 domains and 5 levels of severity each), a measure (EQ-VAS) and a valuation (utility) of health. Multiple linear regression analyses were conducted to assess the association between $E Q-5 D$ VAS score and the utility with age, sex, diagnosis, treatment experience, years from symptoms onset and years from diagnosis.

Results: 513 patients were enrolled (mean $\pm S D=50.0 \pm 13.6,42.5 \%$ female). As regards the diagnosis, $33.9 \%$ had RA, $34.9 \%$ PsA and $31.2 \%$ AS. The mean \pm SD time from the symptoms onset was $10.8 \pm 9.4$ and from the diagnosis was $8.0 \pm 8.2$ years. Almost half of the patients $(47.4 \%)$ were naïve to the biological treatment. Patients reporting severe or extreme problems were: $7.1 \%$ in mobility, $3.6 \%$ in self-care, $10.3 \%$ in usual activities, $18.6 \%$ in pain/discomfort, $5.5 \%$ in anxiety/depression. The mean $\pm S D$ of the VAS was $60.4 \pm 20.5$ and of the utility was $0.773 \pm 0.116$. From the regression model the VAS and utility are significantly $(p<0.05)$ associated with age, sex and treatment experience (table 1). In particular, the patients being naïve to the treatment with biological drugs had on average significant worse levels of $H R Q$ oL than experienced patients, adjusting for the other variables included in the model.

Table 1. Results of the regression analyses

\begin{tabular}{lccccc}
\hline Varibles & \multicolumn{2}{c}{ EQ-5D VAS } & & \multicolumn{2}{c}{ EQ-5D UTILITY } \\
\cline { 2 - 3 } \cline { 5 - 6 } & Coeff. Regr & p-value & & Coeff. regr & p-value \\
\hline Constant & 92.455 & 0.000 & & 0.954 & 0.000 \\
Age & -0.205 & 0.002 & & -0.001 & 0.013 \\
Gender (Female) & -7.473 & 0.000 & & -0.055 & 0.000 \\
Psoriatic arthritis & -3.986 & 0.063 & & -0.009 & 0.448 \\
Ankylosing spondylitis & -4.236 & 0.076 & & -0.016 & 0.228 \\
Naïve patients & -13.873 & 0.000 & & -0.074 & 0.000 \\
Time from symptoms onset & -0.105 & 0.557 & & 0.000 & 0.858 \\
Time from diagnosis & 0.061 & 0.768 & & 0.000 & 0.815 \\
\hline
\end{tabular}

Conclusions: Patients naïve to biological treatment have significat lower levels of $H R Q o L$, suggesting that their current situation is not satisfactory and need to start with a more effective treatment.

Disclosure of Interest: L. Sinigaglia: None declared, L. Scalone: None declared, P. Sarzi-Puttini: None declared, C. Montecucco: None declared, R. Giacomelli Grant/research support from: MSD, G. Lapadula: None declared, I. Olivieri: None declared, A. Giardino Employee of: MSD Italia, G. Didoni Employee of: MSD Italia, P. Cortesi Grant/research support from: Gilead, L. Mantovani: None declared, M. Mecchia Employee of: MSD Italia

DOI: 10.1136/annrheumdis-2017-eular.4904

\section{AB1204-HPR EVALUATION OF CARBOHYDRATE METABOLISM IN RHEUMATOLOGIC PATIENTS AFTER PULSE THERAPY WITH GLUCOCORTICOIDS}

M. Salokhiddinov, A. Tulabaev. Rheumatology, Tashkent Medical Academy, Tashkent, Uzbekistan

Background: Chronic inflammation - the crucial pathogenic mechanism of rheumatoid arthritis is the main cause of accelerated atherosclerosis, insulin resistance and well-known consequences related to it. The conservative treatment of rheumatoid arthritis may provide a significant influence on glucose metabolism. When the duration of rheumatic diseases of administration and dosage of glucocorticoids (GC) are significant predictors of the development of impaired glucose tolerance and diabetes mellitus.

Objectives: To study the effect of pulse-therapy (PT) of the GC on the violation of carbohydrate metabolism in patients with rheumatic diseases

Methods: The study included 35 patients ( 7 men, 18 women) with a variety of rheumatic diseases (systemic lupus erythematosus - 23 , systemic vasculitis - 12) between the ages of 18 to 68 years (mean age $42,3 \pm 14,43$ years) and duration of disease from 6 months to 12 years (mean $3,55 \pm 3,36$ years). Pulse-therapy of GK included intravenous prednisolone $600-1000 \mathrm{mg}$ per day for 3 consecutive days (course dose of 1800-3000 mg). Oral glucose tolerance test (OGTT) was performed after the course. The first group included patients with a normal result of OGTT (glucose concentration of $<7.8 \mathrm{mmol} / \mathrm{L}$ at 2 hours after taking $75 \mathrm{~g}$ of glucose). There were 23 patients in the first group at the age of 18 to 54 years (mean age $38.0 \pm 2.8$ years). Patients of the second group level had OGTT was $>7.8 \mathrm{mmol} / \mathrm{L}$. This group included 12 people aged from 44 to 61 years (mean age $52,6 \pm 8,4$ years). All patients underwent the measurement of blood glucose levels prior to PT, 2, 4, 6, 10, 24, 48 and 72 hours and after the PT

Results: All patients included in the study, after the PT session there was an increase in blood glucose levels with a peak at 4 hours after the start of administration - $12.2 \pm 0.82$ in the first group, in the second to $21.95 \pm 0.25 \mathrm{mmol} / \mathrm{l}$ $(p<0,05)$. Normalization of glucose levels in the first group of patients occurred within $1.75 \pm 0.18$ days ( 1 to 3 ), whereas in the second - for $5,0 \pm 1,0$ (3 to 5 ; $p<0,05)$. During the OGTT the mean fasting blood glucose levels in patients with the first group was $4.49 \pm 0.12 \mathrm{mmol} / \mathrm{l}$, and the second $-5,85 \pm 0,35(p<0,05)$, after 2 hours $-6.0 \pm 0.21$ and $10.0 \pm 1.5 \mathrm{mmol} / \mathrm{l}$, respectively $(p<0,05)$.

Conclusions: Application PT of GC in rheumatic patients causes blood glucose levels to change values, indicating the development of impaired glucose tolerance. Predictors of disorders of carbohydrate metabolism in these patients are high levels of glycemia during the PT more continuous glucose normalization indices after the course PT GC.

References:

[1] Alekseeva El. Pulse therapy with glucocorticoids of systemic manifestations of rheumatoid arthritis in children. Questions of modern times. Pediatrician 2005;4(1)9-14.

[2] Lebets IS, Tolmacheva SR, Shevchenko NS. Modern approaches to the treatment of systemic lupus erythematosus in adolescents. Wedge. Pediatrician 2012;6:91-94

[3] Omelchenko LI, Nikolaenko VB. Some aspects of improving glucocorticoid therapy in rheumatic diseases in children. Ukr. Rheumatology 2010;1:44-49. Disclosure of Interest: None declared DOI: 10.1136/annrheumdis-2017-eular.1169

\section{AB1205-HPR THE EFFECTS OF KINESIOTAPING ON JOINT POSITION SENSE AND POSTURAL STABILITY FOLLOWING FATIGUE PROTOCOL}

O. Aydoğdu, Z. Sarı, U.S. Yurdalan. Physiotherapy and Rehabilitation, Marmara University, Health Sciences Faculty, Istanbul, Turkey

Background: Muscle fatigue is common in sports activities and has been shown to adversely alter proprioception, impair neuromuscular control, and increase the risk of injury. Kinesiotaping has recently gained popularity among sports professionals for its assumed injury prevention and performance enhancement (1). Two studies have reported conflicting findings with respect to the effects of KT on proprioception. Halseth et al reported that KT produced no significant change in the absolute error in ankle joint position sense (2). However, Chang et al reported that KT decreased the force sense error in grip strength measurements among 21 healthy college athletes (3). Thus, the current literature does not provide clear information about the effects of KT on proprioception. Although there are published articles about investigating KT on joint position sense and postural stability, the effects of KT is still unknown after muscle fatigue, to our knowledge.

Objectives: There is a lack of literature examining the KT on joint position sense and postural stability following fatigue protocol. Therefore, the aim of this study was to investigate the effects of KT on knee joint position sense and postural stability after muscle fatigue. It was hypothesized that KT applied on quadriceps femoris muscle would partially compensate for the proprioceptive and balance-related deficits caused by muscle fatigue.

Methods: Thirty - six healthy subjects were evaluated in the study. Knee joint position sense was assessed by Biodex System Pro 4 during active repositioning tests at the target angles of $30^{\circ}, 50^{\circ}$ and $70^{\circ}$ of knee flexion in sagittal plane. Postural stability was assessed by Pedalo Sensamove ${ }^{\circledR}$ System in antero - 